\title{
Neutrino Masses and Mixings: A Theoretical Perspective
}

\author{
Rabindra N. Mohapatra* \\ Dept. of Physics and Maryland Center for Fundamental Physics, University of Maryland, \\ College Park, MD-20742 \\ E-mail: rmohapateumd.edu
}

\begin{abstract}
A brief overview of two directions of research into physics beyond the standard model (SM) that provide an understanding of the recent neutrino mass discoveries is given. One is based on a TeV scale left-right model with many testable predictions for low energy as well as collider experiments e.g. LHC and a second one based on $\mathrm{SO}(10)$ grand unification group with renormalizable interactions only, which is predictive in the neutrino sector. The second one was shown to predict the correct value for $\theta_{13}$ in 2004. Specific baryon number violating processes connected to neutrino mass physics e.g. neutron-anti-neutron oscillation as well as $\mathrm{B}-\mathrm{L}=2$ decay modes of nucleons such as $n \rightarrow e^{-} \pi^{+}$are shown to occur in the SO(10) model at an observable rate.
\end{abstract}

XV Workshop on Neutrino Telescopes,

11-15 March 2013

Venice, Italy

${ }^{*}$ Speaker. 


\section{Introduction}

The discovery of the Higgs boson at the LHC has solved the problem of the origin of mass for the quarks and charged leptons. This is a major accomplishment. There is however a feeling among theorists that the same Higgs field cannot be the source of mass for neutrinos. The reasoning goes as follows: In the standard model (SM), charged fermion masses arise from Yukawa interactions of the Higgs field:

$$
\mathscr{L}_{f}=h_{u} \bar{Q} H u_{R}+h_{d} \bar{Q} \tilde{H} d_{R}+h_{\ell} \bar{L} \tilde{H} \ell_{R}
$$

where $Q, L$ are quark and lepton doublets of SM gauge group and $u_{R}, d_{R}, e_{R}$ are SM singlets. When the SM gauge symmetry is spontaneously broken by $<H^{0}>\neq 0$, we get fermion masses, $m_{f}=$ $h_{f}<H^{0}>$. Since $<H^{0}>$ also gives mass to the gauge bosons, we know that $<H^{0}>=246 \mathrm{GeV}$. Let us use similar argument for the neutrinos. First of all, the standard model has no right handed neutrinos. So to be able to generate Higgs couplings that could give analogous mass terms for neutrinos, we must add a right handed neutrino $v_{R}$ to the standard model. The $v_{R}$ coupling to Higgs field can be written as: $h_{v} \bar{L} H v_{R}$ which after symmetry breaking would lead to $m_{v}=h_{v}\left\langle H^{0}\right\rangle$. However since the observed neutrino oscillation data as well as cosmological data tell us that neutrino masses, $m_{v} \ll \mathrm{eV}$, one would have to set $h_{v} \leq 10^{-12}$ to get such low values. Such small parameters are always problematic from a conceptual point of view leading to the suspicion that there is a new source for neutrino masses other than the standard model $125 \mathrm{GeV}$ Higgs field. In any case, if this was the model for neutrino masses, neutrinos would be Dirac fermions and there would be no way to test the model and experiments searching for neutrino oscillations would simply fix the parameters of the model.

A new possibility for neutrino mass was suggested by Weinberg in 1979. He noted that there could be some new high scale physics that at low energies leads to an effective non-renormalizable operator of the form $\lambda \frac{L H L H}{M}$ [1], where $M$ represents the scale of new physics responsible for this operator. After the SM symmetry breakdown, this operator would lead lead to a Majorana mass $m_{v} \simeq \frac{\left.\lambda<H^{0}\right\rangle^{2}}{M}$ and choosing $M$ appropriately, we can understand why neutrino masses are small. A key question that then arises is: what is the physics behind $M$ ?. The simplest suggestion for this is that $M$ is simply the Majorana mass for the right handed neutrino i.e. $M N^{T} C^{-1} N . N$ being an SM singlet field, such terms can be added to the SM without breaking gauge invariance. There is then a $(v, N)$ mass matrix of the form:

$$
\left(\begin{array}{cc}
0 & m_{D} \\
m_{D}^{\top} & M_{N}
\end{array}\right)
$$

where $m_{D}=h_{v}<H^{0}>$ is the Dirac mass term which mixes the $v$ and $N$ states, and $M_{N}$ is the Majorana mass term for $N$ after diagonalizing this matrix, one gets the formula for light neutrinos $M_{v} \simeq-m_{D} M_{N}^{-1} m_{D}^{\top}$ which is similar to the one given by Weinberg operator. This is known as the seesaw mechanism [2] and searching for the origin of seesaw mechanism can provide significant clue to the nature of new physics beyond the standard model and that behind $M$.

We note that there are several ways to get the Weinberg operator. The above way is known as type I seesaw in the literature. There are other kinds of seesaw mechanisms $[3,4,5]$ with very 
different underlying physics. In the examples below, we will focus only on type I and type II seesaws. In type II seesaw, the particle responsible for the Weinberg operator is a triplet Higgs field with SM hypercharge $Y=2$. A practical and experimental distinction between type I and type II seesaw is that in the former case, there is not only a heavy right handed Majorana neutrino but there is there is also a mixing between the heavy and heavr neutrino given from the seesaw formula as $\xi \simeq \frac{m_{D}}{M_{N}}$, thus causing a departure from the unitarity of the lepton mixing matrix whereas in the type II case, there is no breakdown of unitarity. Searching for the effect of nonvanishing $\xi$ can then be a useful way to look for type I seesaw.

In order to unravel the beyond the standard model physics responsible for the seesaw mechanism, the first thing to note is that there are two key elements to this mechanism: (i) existence of three heavy right handed Majorana neutrinos which means breakdown of the B-L symmetry of standard model and (ii) the scale of their Majorana mass or the scale at which $B-L$ symmetry breaksfor independent reasons. Two classes of theories that naturally provide these two ingredients simultaneously are:

1. Left-right symmetric extensions of the standard model based on the gauge group $S U(2)_{L} \times$ $S U(2)_{R} \times U(1)_{B-L}$ group where three right handed neutrinos are predicted to exist by the requirement of gauge anomaly cancellation and are the parity partner of the familiar neutrinos. Secondly, the seesaw scale turns out to be nothing other than the scale of $S U(2)_{R} \times U(1)_{B-L}$ breaking to the standard model. Thus both the ingredients of seesaw mechanism emerge naturally. The seesaw scale in this case could even be at the $\mathrm{TeV}$, providing hope for testing seesaw mechanism at the Large Hadron Collider (LHC), as we discuss below.

2. $\mathrm{SO}(10)$ grand unified theories, where adding three right handed neutrinos gives 16 -fermions per generation which then fits right into the 16-dimensional spinor representation of the $\mathrm{SO}(10)$ group. Also, this group contains the $B-L$ symmetry as a subgroup ( as in the left-right case) whose breaking leads to the Majorana mass for the right handed neutrinos. Since in simple scenarios, the $B-L$ group breaks at the GUT scale, the seesaw scale in these models is $\sim 10^{16} \mathrm{GeV}$ as would be required by quark-lepton unification.

The above two scenarios focus on the origin of neutrino mass scale. However, there is also the important issue of neutrino mixings which are so different from the quark mixings and a complete theory of neutrino masses should address this issue as well. This is an active field of research and one of the main tools of this research has been to use various discrete flavor symmetries to address this problem. Rightly, there is a large body of literature in this field [6]. Currently the symmetry approach is often discussed within operator framework without any specific gauge model in mind. While a detailed discussion of this approach to neutrino flavor puzzle is beyond the scope of this review, one point worth noting is that generic operator approach with symmetries may not always be compatible with a particular underlying gauge model or in the least may have to respect more constraints. Therefore, if experiments provide evidence for some particular underlying model (either of the two discussed here or some other), the symmetry approach must be embedded within this framework. It may also be that flavor mixing pattern for leptons is a consequence of dynamics as the $\mathrm{SO}(10)$ example below illustrates. Thus it is important to keep an open mind at this stage. 


\section{A "natural" TeV scale left-right model for neutrinos}

Before discussing the details of our model, we review, for completeness, the basic features of generic L-R seesaw models. In its minimal version, the fermions are assigned to the gauge group $S U(2)_{L} \times S U(2)_{R} \times U(1)_{B-L}$ as follows: denoting $Q \equiv(u, d)^{\top}$ and $\psi \equiv\left(v_{\ell}, \ell\right)^{\top}$ as the quark and lepton doublets respectively, $Q_{L}$ and $\psi_{L}$ (also denoted simply by $L$ ) are assigned to doublets under the $S U(2)_{L}$ group, while $Q_{R}$ and $\psi_{R}$ (also denoted by $R$ ) as the doublets under the $S U(2)_{R}$ group. Their $B-L$ quantum numbers can easily be worked out from the definition of the electric charge: $Q=T_{3 L}+T_{3 R}+(B-L) / 2$, where $T_{3 L}$ and $T_{3 R}$ are the third components of isospin under $S U(2)_{L}$ and $S U(2)_{R}$ respectively. The Higgs sector of the model consists of one or several of the following multiplets:

$$
\Delta_{R} \equiv\left(\begin{array}{cc}
\Delta_{R}^{+} / \sqrt{2} & \Delta_{R}^{++} \\
\Delta_{R}^{0} & -\Delta_{R}^{+} / \sqrt{2}
\end{array}\right), \phi \equiv\left(\begin{array}{cc}
\phi_{1}^{0} & \phi_{2}^{+} \\
\phi_{1}^{-} & \phi_{2}^{0}
\end{array}\right) .
$$

The gauge symmetry $S U(2)_{R} \times U(1)_{B-L}$ is broken by the vev $\left\langle\Delta_{R}^{0}\right\rangle=v_{R}$ to the group $U(1)_{Y}$ of the SM. There is also an left handed counterpart, $\Delta_{L}$ to $\Delta_{R}$, which we do not consider here for the following reasons. There are versions of the model where parity and $S U(2)_{R}$ gauge symmetry scales are decoupled so that the $\Delta_{L}$ fields become heavy when the discrete parity symmetry is broken, and disappear from the low energy theory [7]. This version (i.e. LR model without parity) is also necessary to suppress the type II contribution to neutrino mass in low scale left-right models [8], which otherwise could be unacceptably large. The low energy Lagrangian in this case therefore has invariance under the L-R gauge group but not parity. We will focus on this class of models in this paper, which seem to be necessary in implementing our strategy.

In generic models, the vev of the $\phi$ field given by $\langle\phi\rangle=\operatorname{diag}\left(\kappa, \kappa^{\prime}\right)$ breaks the SM gauge group to $U(1)_{\mathrm{em}}$. To see how the fermions pick up mass and how seesaw mechanism arises in the L-R model, we write down the Yukawa Lagrangian of the model:

$$
\begin{aligned}
\mathscr{L}_{Y}= & h_{i j}^{q, a} \bar{Q}_{L, i} \phi_{a} Q_{R, j}+\tilde{h}_{i j}^{q, a} \bar{Q}_{L, i} \tilde{\phi}_{a} Q_{R, j}+h_{i j}^{\ell, a} \bar{L}_{i} \phi_{a} R_{j} \\
& +\tilde{h}_{i j}^{\ell, a} \bar{L}_{i} \tilde{\phi}_{a} R_{j}+f_{i j}\left(R_{i} R_{j} \Delta_{R}+L_{i} L_{j} \Delta_{L}\right)+\text { h.c. },
\end{aligned}
$$

where $i, j$ stand for generations and $a$ for labelling the Higgs bi-doublets, and $\tilde{\phi}=\tau_{2} \phi^{*} \tau_{2}$ ( $\tau_{2}$ being the second Pauli matrix). After symmetry breaking, the Dirac fermion masses are given by the generic formula $M_{f}=h^{f} \kappa+\tilde{h}^{f} \kappa^{\prime}$ for up-type fermions, and for down-type quarks and charged leptons, it is the same formula with $\kappa$ and $\kappa^{\prime}$ interchanged. The Yukawa Lagrangian Eq. (2.2) leads to the Dirac mass matrix for neutrinos $M_{D}=h^{\ell} \kappa+\tilde{h}^{\ell} \kappa^{\prime}$ and the Majorana mass matrix for the heavy RH neutrinos $M_{N}=f v_{R}$ which go into thenseesaw formula for calculating the neutrino masses and the heavy-light neutrino mixing.

As discussed earlier, for generic forms of $M_{D}$ and $M_{N}$, the heavy-light mixing parameter $\xi \simeq \sqrt{M_{v}\left(f v_{R}\right)^{-1}}$ is a tiny number regardless of whether the seesaw scale is in the TeV range or higher. This keeps all heavy-light mixing effects at an unobservable level. In the following section, we present a model with special textures for $M_{D}$ and $M_{N}$ guaranteed by an appropriate discrete symmetry which can lead to "large" heavy-light neutrino mixing with TeV-scale type I seesaw, thereby enhancing the phenomenological richness of the model. 


\subsection{Basic strategy}

The basic strategy for implementing our program is to have the appropriate textures for $M_{D}$ and $M_{N}$ which via type-I seesaw lead to 'large' heavy-light mixing and observed light neutrino masses. There are several examples of this type discussed in the literature $[9,10]$. In this section, we discuss the embedding of one of them (from Ref. [9]) in the L-R model using an appropriate family symmetry. The symmetry must not only guarantee the special leptonic textures but also must be free of light scalar bosons which can result if the effect of the discrete symmetry is to automatically lead to a $U(1)$ symmetry of the full Lagrangian. Moreover in L-R symmetric models, the charged lepton mass matrix can be related to $M_{D}$ which puts additional constraints on phenomenological viability of the model. We therefore find it remarkable that the model we present below remains a viable $\mathrm{TeV}$-scale L-R type I seesaw model for neutrinos, and as a result, has interesting phenomenological implications.

The Dirac and Majorana mass matrices $M_{D}$ and $M_{N}$ considered here have the following form:

$$
M_{D}=\left(\begin{array}{lll}
m_{1} & \delta_{1} & \varepsilon_{1} \\
m_{2} & \delta_{2} & \varepsilon_{2} \\
m_{3} & \delta_{3} & \varepsilon_{3}
\end{array}\right) \text { and } M_{N}=\left(\begin{array}{ccc}
0 & M_{1} & 0 \\
M_{1} & 0 & 0 \\
0 & 0 & M_{2}
\end{array}\right)
$$

with $\varepsilon_{i}, \delta_{i} \ll m_{i}$. In the limit of $\varepsilon_{i}, \delta_{i}$, the neutrino masses vanish, although the heavy-light mixing parameters given by $\xi_{i j} \sim m_{i} / M_{j}$ (roughly speaking) can be quite large. The neutrino masses given by the seesaw formula are dependent upon small parameters $\varepsilon_{i}$ and $\delta_{i}$. If by some symmetry one can guarantee the smallness of $\delta_{i}$ and $\varepsilon_{i}$, then we have an alternative TeV scale seesaw model but with many testable consequences compared to e.g. SM extended with right handed neutrino and a tiny Dirac Yukawa coupling.

The above mass textures were discussed in the context of SM [9]; but as we note below, their embedding into the left-right model leads to very distinct collider as well as low energy signatures. The first challenge is to show that, when these mass textures are embedded into L-R models, they can reproduce the observed neutrino masses and mixing and that any small parameters used can explained. Since in L-R models the charged lepton mass matrix and the Dirac neutrino mass matrix are related, fitting neutrino oscillation data in a manner that also reproduces charged lepton masses is specially nontrivial, in addition to the fact that the seesaw scale is in $\mathrm{TeV}$ range.

\subsection{Naturalness of the special Dirac and Majorana textures}

In order to obtain the special Dirac and Majorana textures given in Eq. (2.3), we use only the L-R gauge group $S U(2)_{L} \times S U(2)_{R} \times U(1)_{B-L}$ without the parity symmetry and supplement it with a global discrete symmetry $D \equiv Z_{4} \times Z_{4} \times Z_{4}$. For the Higgs sector, we choose three bidoublets $\left(\phi_{1,2,3}\right)$ with $B-L=0$ and two RH triplets $\left(\Delta_{R 1, R 2}\right)$ with $B-L=2$. The fermion and Higgs multiplets are assigned the $D$ quantum numbers as shown in Table I below.

The leptonic Yukawa Lagrangian invariant under this symmetry $D$ is given by

$$
\begin{array}{r}
\mathscr{L}_{\ell, Y}=h_{\alpha 1} \bar{L}_{\alpha} \tilde{\phi}_{1} R_{1}+h_{\alpha 2} \bar{L}_{\alpha} \phi_{2} R_{2}+h_{\alpha 3} \bar{L}_{\alpha} \phi_{3} R_{3} \\
f_{12} R_{1} R_{2} \Delta_{R, 1}+f_{33} R_{3} R_{3} \Delta_{R, 2}+\text { h.c. }
\end{array}
$$




\begin{tabular}{c|c}
\hline \hline Field & $Z_{4} \times Z_{4} \times Z_{4}$ transformation \\
\hline$L_{\alpha}$ & $(1,1,1)$ \\
$R_{1}$ & $(-i, 1,1)$ \\
$R_{2}$ & $(1,-i, 1)$ \\
$R_{3}$ & $(1,1,-i)$ \\
$\phi_{1}$ & $(-i, 1,1)$ \\
$\phi_{2}$ & $(1, i, 1)$ \\
$\phi_{3}$ & $(1,1, i)$ \\
$\Delta_{R, 1}$ & $(i, i, 1)$ \\
$\Delta_{R, 2}$ & $(1,1,-1)$ \\
\hline \hline
\end{tabular}

Table 1: The discrete symmetry assignments for the fermion and Higgs fields in our L-R model that lead naturally to the special Dirac and Majorana textures given in Eq. (2.3). Here $\alpha=1,2,3$ for different generations.

An important point to emphasize is that in the discrete symmetry limit, the vevs of $\phi_{1,2,3}$ will have the following form:

$$
\left\langle\phi_{1,2,3}\right\rangle=\left(\begin{array}{cc}
0 & 0 \\
0 & \kappa_{1,2,3}^{\prime}
\end{array}\right) .
$$

Note that there is a range of parameters where this minimum is stable. An easy way to see this is to note that terms of the form $\operatorname{Tr}\left(\tilde{\phi}_{a} \phi_{b}^{\dagger}\right)$ which would change the $\phi$ vev to the form $\operatorname{diag}\left(\kappa, \kappa^{\prime}\right)$ are forbidden by the discrete symmetry from appearing in the scalar potential. So we see that in the symmetry limit, the Dirac mass matrix $M_{D}$ has one column big and non-zero and the charged lepton masses have one eigenvalue zero which we can identify as the electron flavor. The RH neutrino Majorana mass matrix has the form in Eq. (2.3) consistent with this symmetry. To make the model realistic, we add very tiny soft symmetry-breaking terms to the scalar potential as follows:

$$
\delta V(\phi)=\sum_{a, b} \mu_{a b}^{2} \operatorname{Tr}\left(\tilde{\phi}_{a} \phi_{b}^{\dagger}\right)+\text { h.c. }
$$

where $a, b=1,2,3$. Note that $\mu_{a b}^{2}$ are only multiplicatively renormalized. As a result, we can choose their magnitudes to be very small without worrying about radiative destabilization. This will then cause the $\phi_{a}$ vevs to take the following form:

$$
\left\langle\phi_{1,2,3}\right\rangle=\left(\begin{array}{cc}
\delta \kappa_{1,2,3} & 0 \\
0 & \kappa_{1,2,3}^{\prime}
\end{array}\right)
$$

where $\delta \kappa_{a} \propto \mu_{a b}^{2} \kappa_{j}^{\prime} /\left(\sum_{a, b=1}^{3} \lambda \lambda_{a b} \kappa_{a}^{\prime} \kappa_{b}^{\prime}+\lambda v_{R, i}^{2}\right)$. Choosing $\mu_{a b}^{2}$ to be appropriately small, we can get very small $\delta \kappa_{a}$ that we use in the mass fits below. It is possible to generate these small numbers from higher dimensional terms in the potential from a high scale theory that for instance may involve supersymmetry or vector-like quarks etc.

- An important point to notice is that $\delta \kappa_{a}$ are responsible for the electron mass as well as neutrino masses via type I seesaw. Thus getting a fit to the observed neutrino masses and 
mixing while at the same time keeping electron mass at its desired value is a nontrivial task since in the lepton sector, the model has only 11 free parameters and out of this we must not only get fits for the three charged lepton masses, the two neutrino mass-squared differences and three mixing angles (total of 8 outputs), but we must also satisfy the unitarity constraints on the new light neutrino mixing matrix as well as constraints from rare lepton decays which involve only the RH mixing matrix for charged leptons. There are therefore a lot more constraints than inputs and it is shown in [11] that our model does indeed provide a fit to all lepton observables while satisfying all the necessary constraints.

- In typical fits in this kind of models, the $\delta \kappa_{2,3}$ are tiny numbers e.g. $\delta \kappa_{2} \sim 10^{-8} \kappa^{\prime}$ and $\delta \kappa_{3} \sim 10^{-5} \kappa^{\prime}$. These numbers may appear to suggest that there is fine tuning involved; however, we point out that in this model, these are generated by one-loop graphs that induce the terms $\delta \mu^{2} \operatorname{Tr} \phi^{\dagger} \tilde{\phi}$ with $\delta \mu^{2} \sim \frac{g^{2}}{16 \pi^{2}} \delta m_{W_{L}-W_{R}}^{2}$. Estimating $\delta m_{W_{L}-W_{R}}^{2}$, the left-right handed gauge boson mixing induced by the quark sector, predominantly by $t-b$ intermediate states in the loop to be $\delta m_{W_{L}-W_{R}}^{2} \sim \frac{g^{2}}{16 \pi^{2}} m_{t} m_{b} \sim 0.1 \mathrm{GeV}^{2}$, we can expect $\delta \kappa_{a} / \kappa^{\prime} \sim 10^{-7}$ or so making it naturally small. It is interesting to note that the small $\delta \kappa_{a}$ are generated by the physics in the quark sector since that is the only sector that generates $W_{L}-W_{R}$ mixing.

- We also note that in the quark sector, we could have either another bi-doublet field $\phi_{0}$ or some iso-singlet heavy vector-like fields which are singlets under the discrete group $D$ that generate masses for the quarks (which are also assumed to be singlets under $D$ ). In addition to generating to small Dirac masses for neutrinos, the complex Yukawa couplings in this sector can then generate the CKM mixing angles as well as the phase for $C P$ violation in the quark sector.

A second point is that a consequence of the $\left(Z_{4}\right)^{3}$ family symmetry is that our model predicts sub-TeV standard model like heavy Higgs bosons which are leptophilic. This is currently under investigation but the general point worth making is that if LHC limits on SM-like Higgs bosons exceed one $\mathrm{TeV}$ in the leptonic channels, our model will be under serious tension.

Clearly new textures could be generated by putting the column in the Dirac matrix in different places and accordingly choosing the Majorana texture. The experimental signals for such choices will be obviously different and we do not discuss them here.

\subsection{Collider and charged lepton flavor violating signals}

As has been widely discussed $[12,13,14]$, the seesaw mechanism has a clear signal at the LHC in the form of like sign dileptons plus jets $\left(\ell^{ \pm} \ell^{ \pm} j j\right)$ arising from the production of RH neutrinos $\mathrm{N}$ from its mixing (denoted here by $V_{\ell N}$ ) with neutrino from the seesaw mechanism. It turns out that while this is true for most generic textures for $m_{D}$ and $M_{N}$ in the SM extensions to realize seesaw, for the particular textures we have chosen, there is no like sign dilepton signals. However things change drastically, once we choose to embed the textures in Eq. (2.3) into the left-right models. For the specific LR model considered here, for $W_{R}$ mass in the few $\mathrm{TeV}$ range, one can produce a real $W_{R}$, which then can decay to $\mu N_{\mu}$ final state. Depending on the model $N_{\mu}$ can decay in two ways: 
- Via $N_{e} N_{\mu}$ mixing, it can lead to like sign di-lepton states of type $\mu^{ \pm} e^{ \pm}+j j$ if the dominant decay of $N_{e}$ goes via $W_{R}$ exchange;

- Similarly, if the light heavy neutrino mixing is sufficiently "large" due to the structure of $m_{D}$, decays of type $\mu^{ \pm} e^{ \pm}+W_{L}$ final states also predominate [15] . A detailed discussion of this is presented in [15]. The observation of this will probe the Dirac mass more directly.

Coming now to charged lepton flavor violation (CLFV), in the generic TeV scale left-right models, there are two sources of CLFV: one coming from $W_{R}-N_{R}$ exchange and another coming from "large" heavy-light neutrino mixing. In our particular model, the first effect is small but due to the presence of larger $\xi, B(\mu \rightarrow e+\gamma)$ can be as large as $10^{-13}$, which about six times below the current MEG upper limit and can be probed in the near future. For a detailed discussion, see [11].

\section{A minimal SO(10) example}

As noted in the introduction, $S O(10)$ is the minimal GUT that embeds the seesaw paradigm for neutrino masses and among many $\mathrm{SO}(10)$ models in the literature, a class [16] with renormalizable Yukawa couplings appears to be quite predictive without need for extra symmetries. This model uses the Higgs fields 10, 126 and possibly $\mathbf{1 2 0}$ or another 10. If it is a supersymmetric theory, one needs to add an $\mathbf{1 2} 6$ to maintain SUSY down to the TeV scale. there are additional fields that break the GUT symmetry e.g. $\mathbf{4 5}$ and/or $\mathbf{2 1 0}$ but do not contribute to fermion masses. We do not discuss this here.Many of the attractive features of the model relating to fermion masses can be illustrated using the model with one 10-Higgs and one 126-Higgs. Denoting the $\mathbf{1 0}$ Higgs fields ( by $H$ ) and the $\mathbf{1 2 6}+\overline{\mathbf{1 2 6}}$ fields by $\Delta+\bar{\Delta}$, the $S O(10)$ invariant Yukawa couplings of the model can be written as:

$$
\mathscr{L}_{Y}=h_{0} \psi \psi H+f_{0} \psi \psi \bar{\Delta}
$$

where $\psi$ 's denote the $\mathbf{1 6}$ dimensional spinors of $S O(10)$. The Yukawa couplings, $h$ and $f$ are $3 \times 3$ matrices in generation space. This is the most general form of the Yukawa coupling if there is supersymmetry. In the no-supersymmetric case that we focus on here, one can guarantee the absence of other Yukawa couplings by imposing e.g. a Peccei-Quinn symmetry (in which case $\bar{\Delta}=\Delta^{*}$. Both the $\mathbf{1 0}$ and $\mathbf{1 2 6}$ fields contain two standard model doublets each and once electroweak symmetry is broken, they lead to masses for charged fermions and Dirac mass for the neutrinos as follows:

$$
\begin{aligned}
M_{u} & =h+r_{2} f, \\
M_{d} & =\frac{r_{1}}{\tan \beta}(h+f), \\
M_{\ell} & =\frac{r_{1}}{\tan \beta}(h-3 f), \\
M_{v^{D}} & =h-3 r_{2} f .
\end{aligned}
$$

where we have absorbed some of the vev of Higgs doublets into $h, f, h^{\prime}$ and $r_{1,2}$ are the ratios of the other vevs [16]. The neutrino mass formula is a combination of a type I and type II term:

$$
M_{v}=f v_{L}-M_{v^{D}} \frac{1}{f v_{B L}} M_{v^{D}}
$$


This model was analysed with type I seesaw in [17]. It was subsequently pointed out that if the type II term in the neutrino mass dominates, there is a relation between neutrino and charged fermion mass matrices:

$$
M_{v}=c\left(M_{d}-M_{\ell}\right)
$$

and this formula combined with $b-\tau$ mass equality at GUT scale from RGER extrapolation, provides a natural explanation of large atmospheric neutrino mixing angle [18] for the case of two generations. Soon afterwards, it was noted in [19] that once this idea is extended to three generations, it not only explains the large solar angle but it also explains the relation $\frac{m_{\text {solar }}}{m_{\text {atmos }}} \sim$ $\theta_{\text {Cabibbo }}$. Furthermore, it leads to a value for $\theta_{13} \sim \theta_{\text {Cabibbo }} \sim 9-10^{0}$ (for the case when $h^{\prime}=0$ ). These results are extremely interesting since without any symmetries, the simple fact of $b-\tau$ unification seems to qualitatively reproduce all observations. To see how this comes about, observe that $M_{d}$ and $M_{\ell}$ are both hierarchical mass matrices- the former due to small CKM angles and latter due to the constraint of $S O(10)$ grand unification in 3.2. Thus one can write

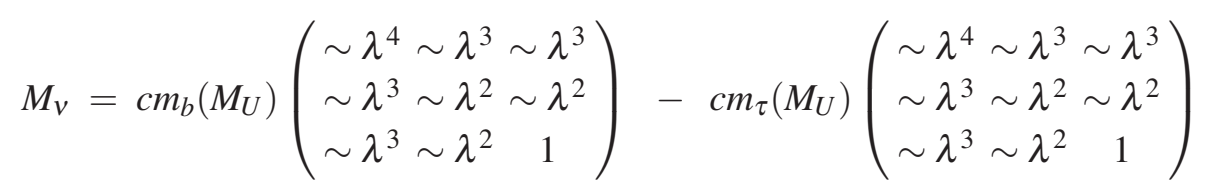

where $\lambda \sim 0.22$, the Cabibbo angle. Note that at GUT scale if $m_{b}\left(M_{U}\right) \sim m_{\tau}\left(M_{U}\right)\left(1+\lambda^{2}\right)$, the $3 \times 3$ entries cancel out in the above difference and one naturally gets for the neutrino mass matrix:

$$
M_{v}=\operatorname{cm}_{\tau}\left(M_{U}\right) \lambda^{2}\left(\begin{array}{ccc}
\sim \lambda^{2} & \sim \lambda & \sim \lambda \\
\sim \lambda & 1+\lambda & 1 \\
\sim \lambda & 1 & 1
\end{array}\right)
$$

where we have omitted order one coefficients in the matrix elements. This mass matrix for neutrinos clearly implies that both the solar and the atmospheric mixing angles are large and $\frac{m_{\text {solar }}}{m_{\text {atmos }}} \sim \lambda$ and also $\theta_{13} \sim \lambda$ [19]. Furthermore note that without $\mathrm{CP}$ violation, the model has only eleven parameters and yet it fits quark and lepton masses and mixings as well as neutrino mixings and masses so well. This model has been the subject of extensive analysis [20].

Extensions of this model by the addition of a $\mathbf{1 2 0}$ Higgs multiplet was then considered and shown to considerably broaden the parameter space while still preserving the "large" $\theta_{13}$ prediction. Recall that 10,126 and $\mathbf{1 2 0}$ are the only three irreducible representations that appear in the product of two spinors of $\mathrm{SO}(10)$; so in some sense it was a natural thing to consider. A particularly interesting version of the $10+126+120$ model is one that assumes that $\mathrm{CP}$ is a good symmetry prior to spontaneous breaking by the vev of the $\mathbf{1 2 0}$ field [21], which is assumed to be odd under under CP. Since $\mathbf{1 2 0}$ gives anti-symmetric Yukawa couplings, its CP odd property after symmetry breaking hermitian fermion mass matrices, responsible for $\mathrm{CP}$ violation both in the quark and lepton sectors. As a result, CKM phase and the Dirac phase of the neutrinos get connected. The model has a total of 16 parameters in the fermion mass sector (12 from Yukawa couplings and four from the vevs) keeping the model still predictive. Three inputs from the neutrino sector e.g. solar and atmospheric mass differences and the atmospheric mixing angle in addition to thirteen from the charged fermion sector allow one to predict $\theta_{12}, \theta_{13}$ and the neutrino CP phases. 


\section{Testing $S O(10)$ grand unification of seesaw via $B$-violating operators}

Testing seesaw idea is often difficult in GUT theories without supersymmetry due to the fact that seesaw scale is very high (close to the GUT scale). In the presence of supersymmetry, there are observable CLFV effects e.g. $B(\mu \rightarrow e+\gamma)$ etc. The only way to test non-susy seesaw models appears to be in the area of baryon number violation. Here however, it is worth pointing out that the canonical B-L conserving modes e.g. $p \rightarrow e^{+} \pi^{0}$ are not directly connected to seesaw physics. So there observation, while very important to test minimal GUT models e.g. $\mathrm{SO}(10)$ do not directly make any statement about seesaw mechanism. It was recently pointed out [22] that in minimal nonsusy as well susy $\mathrm{SO}(10)$ models with 126 Higgs fields, there exist B-L violating nucleon decay models directly connected to the seesaw mechanism. We discuss this below.

It was shown in [22] that there are $d=7$ baryon number violating operators that arise naturally in the context of $S O(10)$ unified theories after the spontaneous breaking of $(B-L)$ needed for seesaw mechanism. A variety of scenarios can emerge. For instance, $(B-L)$ symmetry may break at the GUT scale so that $S O(10)$ breaks directly to the Standard Model gauge symmetry, or it may break at an intermediate scale $M_{I}$ below the GUT scale. In the latter case the intermediate symmetry could be one among several possibilities: $S U(4)_{C} \times S U(2)_{L} \times S U(2)_{R} ; S U(4)_{C} \times S U(2)_{L} \times U(1)_{R}$; $S U(3)_{C} \times S U(2)_{L} \times S U(2)_{R} \times U(1)_{B-L} ; S U(3)_{C} \times S U(2)_{L} \times U(1)_{R} \times U(1)_{B-L} ;$ or $S U(5) \times U(1)$, with or without left-right parity symmetry. In the non-supersymmetric version an intermediate scale is necessary to be compatible with gauge coupling unification[8], while with supersymmetry the direct breaking of $S O(10)$ down to the MSSM is preferable. Even in the latter case, there is room for intermediate scale particles, provided that they form complete multiplets of the $S U(5)$ subgroup, since such particles do not spoil the unification of gauge couplings observed with the MSSM spectrum.

To give an example of how new nucleon decay modes connected to neutrino mass arise within $S O(10)$, we write down the Yukawa couplings in the most general setup [22]. The Higgs fields which can couple to the fermion bi-linears $16_{i} 16_{j}$ are $10_{H}, \overline{126}_{H}$ and $120_{H}$, with the couplings of the $10_{H}$ and $\overline{126}_{H}$ being symmetric in flavor indices $(i, j)$ and those of the $120_{H}$ being antisymmetric. The terms in these Yukawa couplings that are relevant for our discussion are:

$$
\begin{aligned}
\mathscr{L}\left(16_{i} 16_{j} 10_{H}\right)= & h_{i j}\left[\left(u_{i}^{c} Q_{j}+v_{i}^{c} L_{j}\right) h-\left(d_{i}^{c} Q_{j}+e_{i}^{c} L_{j}\right) \bar{h}+\left(\frac{\varepsilon}{2} Q_{i} Q_{j}+u_{i}^{c} e_{j}^{c}-d_{i}^{c} v_{j}^{c}\right) \omega\right. \\
& \left.+\left(\varepsilon u_{i}^{c} d_{j}^{c}+Q_{i} L_{j}\right) \omega^{c}\right] \\
\mathscr{L}\left(16_{i} 16_{j} \overline{\left.126_{H}\right)}=\right. & f_{i j}\left[\left(u_{i}^{c} Q_{j}-3 v_{i}^{c} L_{j}\right) h-\left(d_{i}^{c} Q_{j}-3 e_{i}^{c} L_{j}\right) \bar{h}\right. \\
& +\sqrt{3} i\left(\frac{\varepsilon}{2} Q_{i} Q_{j}-u_{i}^{c} e_{j}^{c}+v_{i}^{c} d_{j}^{c}\right) \omega_{1}+\sqrt{3} i\left(Q_{i} L_{j}-\varepsilon u_{i}^{c} d_{j}^{c}\right) \omega_{1}^{c} \\
& +\sqrt{6}\left(d_{i}^{c} v_{j}^{c}+u_{i}^{c} e_{j}^{c}\right) \omega_{2}+2 \sqrt{3} i d_{i}^{c} L_{j} \rho-2 \sqrt{3} i v_{i}^{c} Q_{j} \bar{\rho}+2 \sqrt{3} u_{i}^{c} v_{j}^{c} \eta \\
& \left.-2 \sqrt{3} i u_{i}^{c} L_{j} \chi+2 \sqrt{3} i e_{i}^{c} Q_{j} \bar{\chi}-2 \sqrt{3} d_{i}^{c} e_{j}^{c} \delta+\sqrt{6} i Q_{i} L_{j} \bar{\Phi}+\ldots\right], \\
\mathscr{L}\left(16_{i} 16_{j} 120_{H}\right)=g_{i j} & {\left[\left(d_{i} Q^{j}+e_{i}^{c} L_{j}\right) \bar{h}_{1}-\left(u_{i}^{c} Q_{j}+v_{i}^{c} L_{j}\right) h_{1}-\sqrt{2} Q_{i} L_{j} \omega_{1}^{c}\right.} \\
- & \sqrt{2}\left(u_{i}^{c} e_{j}^{c}-d_{i}^{c} v_{j}^{c}\right) \omega_{1}-\frac{i}{\sqrt{3}}\left(d_{i}^{c} Q_{j}-3 e_{i}^{c} L_{j}\right) \bar{h}_{2}+\frac{i}{\sqrt{3}}\left(u_{i}^{c} Q_{j}-3 v_{i}^{c} L_{j}\right) h_{2}
\end{aligned}
$$




$$
\begin{aligned}
& -2 e_{i}^{c} Q_{j} \bar{\chi}+2 v_{i}^{c} Q_{j} \bar{\rho}-2 d_{i}^{c} L_{j} \rho+2 u_{i}^{c} L_{j} \chi \\
& -i \varepsilon d_{i}^{c} d_{j}^{c} \bar{\eta}+2 i u_{i}^{c} v_{j}^{c} \eta+\sqrt{2} i \varepsilon d_{i}^{c} u_{j}^{c} \omega_{2}^{c}+\sqrt{2} i\left(d_{i}^{c} v_{j}^{c}-e_{i}^{c} u_{j}^{c}\right) \omega_{2} \\
& \left.-\frac{\varepsilon}{\sqrt{2}} Q_{i} Q_{j} \Phi-\sqrt{2} Q_{i} L_{j} \bar{\Phi}-2 i d_{i}^{c} e_{j}^{c} \delta+i \varepsilon u_{i}^{c} u_{j}^{c} \bar{\delta}+\ldots\right] .
\end{aligned}
$$

These terms are written in terms of the Standard Model decomposition of the sub-multiplets. $\varepsilon$ stands for the $S U(3)_{C}$ tensor $\varepsilon_{\alpha \beta \gamma}$. Here we have not displayed terms that are irrelevant for inducing the $d=7$ baryon number violating operators. (Specifically, we have omitted color singlet, color octet, and color sextet couplings.) The Yukawa couplings obey $h_{i j}=h_{j i}, f_{i j}=f_{j i}$ and $g_{i j}=-g_{j i}$. The $S U(3)_{C} \times S U(2)_{L} \times U(1)_{Y}$ quantum numbers of the various sub-multiplets are given as follows.

$$
\begin{array}{llll}
h(1,2,+1 / 2), & \bar{h}(1,2,-1 / 2), & \omega(3,1,-1 / 3), & \omega^{c}(\overline{3}, 1,1 / 3), \\
\rho(3,2,1 / 6), & \bar{\rho}(\overline{3}, 2,-1 / 6), & \eta(3,1,2 / 3), & \bar{\eta}(\overline{3}, 1,-2 / 3), \\
\Phi(3,3,-1 / 3), & \bar{\Phi}(\overline{3}, 3,1 / 3), & \chi(3,2,7 / 6), & \bar{\chi}(\overline{3}, 2,-7 / 6), \\
\delta(3,1,-4 / 3), & \bar{\delta}(\overline{3}, 1,4 / 3) . &
\end{array}
$$

Different fields with the same SM quantum numbers appear in some couplings, they are distinguished by subscripts 1,2 etc. We have used the same notation for fields with the same SM quantum numbers in $10_{H}, \overline{126}_{H}$ and $120_{H}$, but it should be understood that these are distinct fields. After GUT symmetry breaking various subfields with the same SM quantum number would mix. Some of these mixings would involve the vacuum expectation value of the SM singlet field from the $\overline{126}_{H}$, denoted by $\Delta^{c}$ carrying $(B-L)=-2$. It is this field that supplies large Majorana mass for the right-handed neutrino through the coupling $f_{i j} \sqrt{6} v_{i}^{c} v_{j}^{c} \Delta^{c}$. With $\left\langle\Delta^{c}\right\rangle \neq 0$, trilinear scalar couplings of the type $\rho^{*} \omega H, \eta^{*} \rho H, \rho^{*} \Phi H$ and $\chi^{*} \eta H$, will develop. This issue has been discussed in more detail in [22], but we note that such couplings are invariant under the unbroken SM gauge symmetry. When combined with the Yukawa couplings of Eqs. (4.1)-(4.3), they would induce baryon number violating decays e.g. $n \rightarrow e^{-} \pi^{+}$via the diagram in Fig. 1 .

\subsection{Scenario with observable neutron-anti-neutron oscillation}

In an alternative minimal $\mathrm{SO}(10)$ scenario, we break the $\mathrm{SO}(10)$ group down to the standard model at GUT scale. However, in this case to retain coupling unification, one can have color sextet fields $\Delta_{u^{c} d^{c}}$ along with two $S U(2)_{L}$ triplets with $Y=0$ at the TeV scale. This model leads to $n-\bar{n}$ oscillation via the diagram in Fig. 2 [24]. Due to the TeV scale diquark intermediate state in Fig 2 , this diagram leads to an $n-\bar{n}$ transition rate near $10^{10} \mathrm{sec}$.

\section{Conclusion}

While the observation of neutrino masses and mixings clearly require new physics beyond the standard model, the specific direction of this new physics is certainly very far from clear. If one accepts the seesaw mechanism as then way to understand small neutrino masses, at two natural candidates present themselves. The first one is the left-right symmetric theory whose scale can be in the $\mathrm{TeV}$ range leading to plethora of collider and low energy tests. We have presented an example of such a scenario which has the attractive feature that small parameters used to understand small 

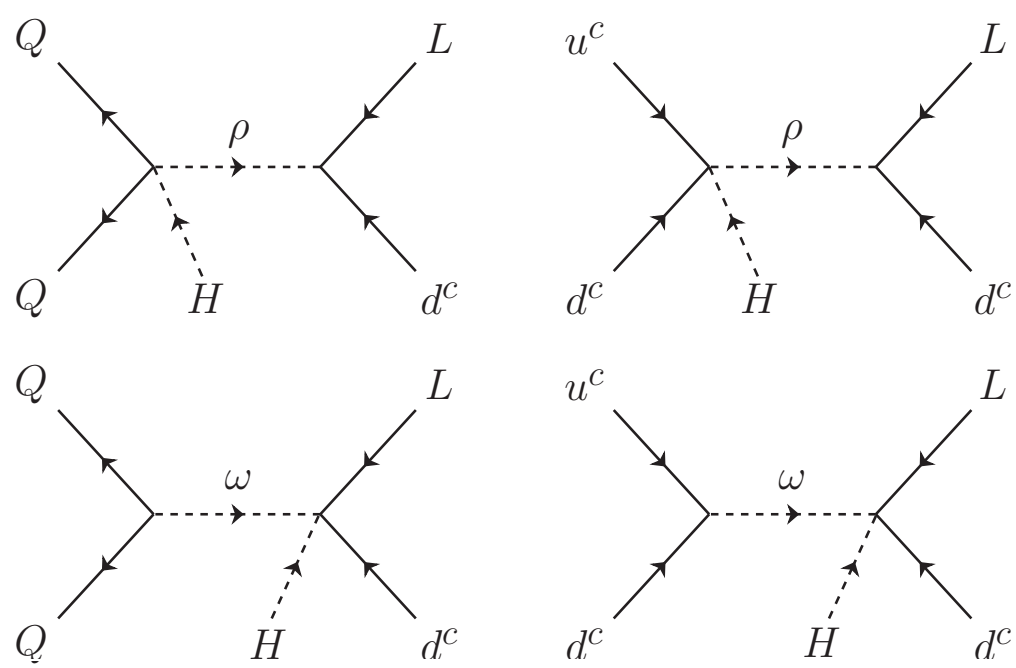

Figure 1: Effective baryon number violating process $n \rightarrow e^{-} \pi^{+}$induced by the symmetric Yukawa couplings of $10_{H}$ and $\overline{126}_{H}$ of $S O(10)$. Here the SM quantum numbers of the various fields are $\omega(3,1,-1 / 3), \rho(3,2,1 / 6)$, and $H(1,2,1 / 2)$.

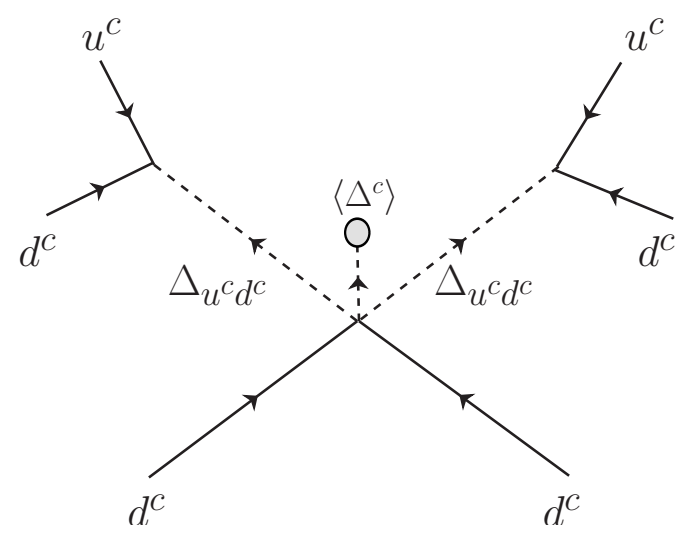

Figure 2: Effective $\Delta B=2$ baryon number violating operator that leads to $n-\bar{n}$ oscillation, induced by the symmetric Yukawa couplings of $10_{H}$ and $\overline{126}_{H}$ of $S O(10)$. Here the SM quantum numbers of the various fields are $\omega(3,1,-1 / 3), \rho(3,2,1 / 6)$, and $H(1,2,1 / 2)$.

neutrino masses have a symmetry protection as well as radiative origin. This model treats quarks and leptons necessarily separately with quark sector physics explaining the small parameters in neutrino sector. On the other hand if we want to consider a quark-lepton unified framework, the natural and minimal framework is an $\mathrm{SO}(10)$ model where then seesaw scale generally is at the GUT scale. In particular, if one uses only renormalizable couplings for fermion masses, the model remains predictive and is testable by searches for $B-L$ violating nucleon decays as well as neutronanti-neutron oscillation, both of which can occur at observable rates consistent with the requirement of grand unification. Some salient features of these two theories are outlined in this talk. 


\section{Acknowledgement}

This work was supported by the National Science Foundation Grant Number PHY-0968854. I would like to thank K. S. Babu, P. S. B. Dev and C. H. Lee for collaboration on different aspects of the models discussed in this paper.

\section{References}

[1] S. Weinberg, Phys. Rev. Lett. 43, 1566 (1979).

[2] P. Minkowski, Phys. Lett. B 67 , 421 (1977); R. N. Mohapatra and G. Senjanovic, Phys. Rev. Lett, 44 , 912 (1980); T. Yanagida in Workshop on Unified Theories, KEK Report 79-18, p. 95, 1979;

M. Gell-Mann, P. Ramond and R. Slansky, Supergravity, p. 315. Amsterdam: North Holland, 1979; S. L. Glashow, 1979 Cargese Summer Institute on Quarks and Leptons, p. 687. New York: Plenum, 1980.

[3] G. Lazarides, Q. Shafi and C. Wetterich, Nucl. Phys. B 181, 287 (1981); J. Schechter and J. W. F. Valle, Phys. Rev. D 22, 2227 (1980); R. N. Mohapatra and G. Senjanovic, Phys. Rev. D 23, 165 (1981).

[4] R. Foot, H. Lew, X. G. He and G. C. Joshi, Z. Phys. C 44, 441 (1989); E. Ma and D. P. Roy, Nucl. Phys. B 644, 290 (2002)

[5] R. N. Mohapatra, Phys. Rev. Lett. 56, 561 (1986); R. N. Mohapatra and J. W. F. Valle, Phys. Rev. D 34, 1642 (1986).

[6] For reviews of the symmetry approach to neutrino flavor, see G. Altarelli and F. Feruglio, Rev. Mod. Phys. 82, 2701 (2010); S. F. King and C. Luhn, Rept. Prog. Phys. 76, 056201 (2013).

[7] D. Chang, R. N. Mohapatra and M. K. Parida, Phys. Rev. Lett. 52, 1072 (1984).

[8] D. Chang and R. N. Mohapatra, Phys. Rev. D 32, 1248 (1985).

[9] J. Kersten and A. Y. Smirnov, Phys. Rev. D 76, 073005 (2007) [arXiv:0705.3221 [hep-ph]].

[10] A. Pilaftsis, Z. Phys. C 55, 275 (1992) [hep-ph/9901206]; J. Gluza, Acta Phys. Polon. B 33, 1735 (2002) K. Kiers, M. Assis, D. Simons, A. A. Petrov and A. Soni, Phys. Rev. D 73, 033009 (2006) Z. -z. Xing, Prog. Theor. Phys. Suppl. 180, 112 (2009) X. -G. He, S. Oh, J. Tandean and C. -C. Wen, Phys. Rev. D 80, 073012 (2009)A. Ibarra, E. Molinaro and S. T. Petcov, JHEP 1009, 108 (2010) M. Mitra, G. Senjanovic and F. Vissani, Nucl. Phys. B 856, 26 (2012) N. Haba, T. Horita, K. Kaneta and Y. Mimura, arXiv:1110.2252 [hep-ph].

[11] P. S. B. Dev, C. -H. Lee and R. N. Mohapatra, arXiv:1309.0774 [hep-ph].

[12] W.-Y. Keung and G. Senjanović, Phys. Rev. Lett. 50, 1427 (1983).

[13] F. del Aguila and J. A. Aguilar-Saavedra, Nucl. Phys. B 813, 22 (2009).

[14] T. Han, I. Lewis, R. Ruiz and Z. -g. Si, Phys. Rev. D 87, 035011 (2013) [Erratum-ibid. D 87, no. 3, 039906 (2013)].

[15] C. -Y. Chen, P. S. B. Dev and R. N. Mohapatra, Phys. Rev. D 88, 033014 (2013) [arXiv:1306.2342 [hep-ph]].

[16] K. S. Babu, R. N. Mohapatra, Phys. Rev. Lett. 70, 2845 (1993).

[17] T. Fukuyama and N. Okada, JHEP 0211, 011 (2002); 
[18] B. Bajc, G. Senjanovic and F. Vissani, hep-ph/0110310; Phys. Rev. Lett. 90, 051802 (2003);

[19] H. S. Goh, R. N. Mohapatra, S. P. Ng, Phys. Lett. B570, 215 (2003); K.S. Babu, C. Macesanu, Phys. Rev. D72, 115003 (2005); S. Bertolini, T. Schwetz, M. Malinsky, Phys. Rev. D73, 115012 (2006); A. S. Joshipura, K. M. Patel, arXiv:1105.5943 [hep-ph];

[20] C. S. Aulakh, B. Bajc, A. Melfo, G. Senjanovic, F. Vissani, Phys. Lett. B588, 196-202 (2004);

T. Fukuyama, A. Ilakovac, T. Kikuchi, S. Meljanac, N. Okada, Phys. Rev. D72, 051701 (2005);

C. S. Aulakh and S. K. Garg, Nucl. Phys. B 857, 101 (2012).

[21] B. Dutta, Y. Mimura and R. N. Mohapatra, Phys. Rev. D 72, 075009 (2005).

[22] K. S. Babu and R. N. Mohapatra, Phys. Rev. Lett. 109, 091803 (2012); Phys.Rev. D86, 035018 (2012).

[23] D. Chang, R. N. Mohapatra, J. Gipson, R. E. Marshak and M. K. Parida, Phys. Rev. D 31, 1718 (1985).

[24] K. S. Babu and R. N. Mohapatra, Phys. Lett. B 715, 328 (2012). 